\title{
Effects of magnetic dilution in the ferrimagnetic columnar ordered $\mathrm{Sm}_{2} \mathrm{MnMnMn} \mathbf{H n}_{-x} \mathrm{Ti}_{x} \mathrm{O}_{12}$ perovskites
}

\author{
Anuradha M. Vibhakar $\odot,{ }^{1,2, *}$ Dmitry D. Khalyavin, ${ }^{3}$ Pascal Manuel, ${ }^{3}$ Ran Liu $\odot, 4,5$ \\ Kazunari Yamaura, ${ }^{4,5}$ Alexei A. Belik ${ }^{\circ},{ }^{4}$ and Roger D. Johnson ${ }^{1,2}$ \\ ${ }^{1}$ Clarendon Laboratory, Department of Physics, University of Oxford, Oxford OX1 3PU, United Kingdom \\ ${ }^{2}$ Department of Physics and Astronomy, University College London, Gower Street, London WC1E 6BT, United Kingdom \\ ${ }^{3}$ ISIS Facility, Rutherford Appleton Laboratory-STFC, Chilton, Didcot OX11 OQX, United Kingdom \\ ${ }^{4}$ International Center for Materials Nanoarchitectonics (WPI-MANA), National Institute for Materials Science (NIMS), \\ Namiki 1-1, Tsukuba, Ibaraki 305-0044, Japan \\ ${ }^{5}$ Graduate School of Chemical Sciences and Engineering, Hokkaido University, North 10 West 8, Kita-ku, \\ Sapporo, Hokkaido 060-0810, Japan
}

(Received 22 September 2020; accepted 7 December 2020; published 22 December 2020)

\begin{abstract}
Powder neutron-diffraction experiments have been employed to establish the effects of site-selective magnetic dilution in the $\mathrm{Sm}_{2} \mathrm{MnMnMn}_{4-x} \mathrm{Ti}_{x} \mathrm{O}_{12} A$-site columnar ordered quadruple perovskite manganites $(x=1, x=2$, and $x=3$ ). We show that in all three compositions the Mn ions adopt a collinear ferrimagnetic structure below 27,62 , and $34 \mathrm{~K}$, respectively. An unexpected increase in the ordering temperature was observed between the $x=1$ and $x=2$ samples, which indicates a considerable departure from mean-field behavior. This result is corroborated by large reductions in the theoretical ground-state magnetic moments observed across the series, which indicate the presence of spin fluctuations and/or disorder. We show that long-range magnetic order in the $x=3$ sample, which occurs below the percolation threshold for $B-B$ exchange, can only be understood to arise if it is mediated via both $A-B$ and $B-B$ exchange, hence confirming the importance of $A-B$ exchange interactions in these materials. Finally, we show that site-selective magnetic dilution enables the tuning of a ferrimagnetic compensation point and the introduction of temperature-induced magnetization reversal.
\end{abstract}

DOI: 10.1103/PhysRevB.102.214428

\section{INTRODUCTION}

The simple perovskite manganites (general chemical formula $A B \mathrm{O}_{3}, B=\mathrm{Mn}$ ) are canonical examples of correlated electron systems in which charge, spin, and orbital order can be tuned to give rise to exotic electronic ground states. This is most famously demonstrated in $\mathrm{La}_{1-x} A_{x}^{\prime} \mathrm{MnO}_{3}\left(A^{\prime}=\right.$ $\mathrm{Ca}^{2+}, \mathrm{Sr}^{2+}$, and $\mathrm{Ba}^{2+}$ ), where a transition from an antiferromagnetic insulating phase to a ferromagnetic metallic phase, driven by dopant concentration, leads to the emergence of the technologically important property of colossal magnetoresistance [1]. More broadly, when the $A$ site is occupied by a rare-earth ion or yttrium $(R)$, a variety of $B$-site magnetic structures have been found. For example, $A$-type antiferromagnetic order was observed experimentally for $R=\mathrm{La} \rightarrow \mathrm{Gd}[2,3]$, and for compositions with smaller $R^{3+}$ ionic radii $(\mathrm{Ho} \rightarrow \mathrm{Yb}), E$-type antiferromagnetic order is stabilized [3]. Furthermore, in the mixed-valence manganites, such as $\mathrm{La}_{0.5} \mathrm{Ca}_{0.5} \mathrm{MnO}_{3}$, zigzag spin chains of the magnetic structure of CE type arise as a result of the charge and orbital order associated with a checkerboard arrangement of $B$-site $\mathrm{Mn}^{3+}$ and $\mathrm{Mn}^{4+}$ ions [4]. Despite considerable departures from the ideal $180^{\circ} \mathrm{Mn}-\mathrm{O}-\mathrm{Mn}$ bonding geometry of up to $40^{\circ}$ (due to octahedral tilts), these magnetic structures can be well understood in terms of dominant $B-B$ magnetic exchange interactions described by the Goodenough-Kanamori-Anderson

*Corresponding author: Anuradha.vibhakar@physics.ox.ac.uk
(GKA) rules [5]. Rare-earth magnetism can play an important role via $A-A$ and $A-B$ interactions, but typically only at low temperatures [6] owing to the much weaker exchange between $f-f$ and $f-d$ electrons, respectively, compared with that of $d-d$ electrons.

In the $A \mathrm{Mn}_{7} \mathrm{O}_{12}$ quadruple perovskite manganites a large $a^{+} a^{+} a^{+}$octahedral tilting pattern (in Glazer notation [7]) introduces an ordered arrangement of Mn ions onto the $A$ sites of the perovskite framework such that these systems also incorporate $A-A$ and $A-B d-d$ exchange pathways. Compared with the simple perovskites, competition between $B-B$ exchange and these additional interactions can lead to new and complex paradigms in frustrated magnetism, especially because the $B-B$ exchange is diminished due to the large octahedral tilting pattern (Mn-O-Mn bond angles reduced by $\sim 40-45^{\circ}$ away from the ideal $\left.180^{\circ}[8,9]\right)$. For example, when $R=\mathrm{La}, \mathrm{Ce}, \mathrm{Nd}, \mathrm{Sm}$, and Eu a collinear ferrimagnetic structure was observed that cannot be explained by dominant $B-B$ interactions alone [10,11]. For $A=\mathrm{Ca}, \mathrm{Sr}, \mathrm{Cd}$, and $\mathrm{Pb}$ and $\mathrm{Na}_{1-x} \mathrm{Ca}_{x} \mathrm{Mn}_{7} \mathrm{O}_{12}$, more complex magnetic structures were stabilized: a constant moment magnetic helix with a modulated spin helicity and an incommensurate pseudo-CE-type phase, respectively [12-14]. Both incommensurate structures arise from a balance between competing $A-A, A-B$, and $B-B$ exchange interactions.

Recently, a family of $A_{2} A^{\prime} A^{\prime \prime} B_{4} \mathrm{O}_{12} \quad A$-site columnar ordered quadruple perovskite manganites have been synthesized [15]. In these manganese oxides a large $a^{+} a^{+} c^{-}$ 
octahedral tilting pattern gives rise to three crystallographically distinct $A$ sites and creates a unique set of exchange pathways not found in either the simple or quadruple perovskite manganites described above. Here, both $A-B$ and $B-B d-d$ superexchange interactions are present, and the $a^{+} a^{+} c^{-}$tilts remove $A-A d-d$ superexchange pathways, which are then reduced to super-superexchange. The weakness of $A-A d-d$ exchange has recently been demonstrated in (NaDy)MnMnTi ${ }_{4} \mathrm{O}_{12}$ [16], where in the absence of $A-B$ and $B$ - $B$ interactions $\left(B=\mathrm{Ti}^{4+}\right)$, antiferromagnetic order develops on the $A$-site sublattices only below $12 \mathrm{~K}$. To the contrary in $\mathrm{Tm}_{2} \mathrm{MnMnMn}_{4} \mathrm{O}_{12}$, the presence of both $A-B$ and $B-B$ exchange stabilizes ferrimagnetic order below $74 \mathrm{~K}$, with a ferromagnetic $B$-site sublattice that is in direct contradiction to the $C$-type antiferromagnetic $B$-site sublattice theoretically predicted by $B-B$ exchange alone - a state that is instead thought to arise as a result of $A-B$ exchange dominating over both $A-A$ and $B-B$ exchange [17]. Indeed, it was shown that when $A-B$ exchange is weakened by substituting $\mathrm{Cu}^{2+}$ for $\mathrm{Mn}^{3+}$ on the $A^{\prime}$ sites $\left(R_{2} \mathrm{CuMnMn}_{4} \mathrm{O}_{12}, R=\mathrm{Dy}\right.$ and $\left.\mathrm{Y}\right)$, antiferromagnetic spin canting is introduced onto the $B$-site sublattice [18]. Hence it has become clear that the $A$-site columnar ordered quadruple perovskite manganites present a flexible framework in which novel frustrated geometries of $A-A, A-B$, and $B-B$ exchange interactions may be tuned through chemical substitution to give rise to unconventional magnetic states.

In this paper, we report the tuning of magnetic exchange interactions in the $\mathrm{Sm}_{2} \mathrm{MnMnMn}_{4-x} \mathrm{Ti}_{x} \mathrm{O}_{12} A$-site columnar ordered quadruple perovskite manganites by chemical substitution of nonmagnetic $\mathrm{Ti}^{4+}$ for $B$-site $\mathrm{Mn}^{3+}$ for $x=1, x=2$, and $x=3$. This series interpolates between the $A$-site-only, antiferromagnetic structure of $(\mathrm{NaDy}) \mathrm{MnMnTi}_{4} \mathrm{O}_{12}[16]$ and the full ferrimagnetic structure of $\mathrm{Tm}_{2} \mathrm{MnMnMn}_{4} \mathrm{O}_{12}$ [17], discussed above. $\mathrm{Sm}_{2} \mathrm{MnMnMn}_{4-x} \mathrm{Ti}_{x} \mathrm{O}_{12}$ has a tetragonal crystal structure with space group $P 4_{2} / n m c$, where the charge and orbital order responsible for the lower orthorhombic symmetry of the $R_{2} \mathrm{MnMnMn}_{4} \mathrm{O}_{12}$ manganites $[15,17]$ has been suppressed by the $B$-site chemical disorder [19]. The three symmetry-inequivalent $A$ sites, shown in Fig. 1(a), labeled $A$ (Sm1), $A^{\prime}(\mathrm{Mn} 1)$, and $A^{\prime \prime}(\mathrm{Mn} 2)$, are occupied by tenfold coordinated $\mathrm{Sm}^{3+}$, by square-planar coordinated $\mathrm{Mn}^{2+}$ or $\mathrm{Mn}^{3+}$, depending on the amount of $\mathrm{Ti}^{4+}$ substituted on the $B$ sites, and by tetrahedrally coordinated $\mathrm{Mn}^{2+}$, respectively. The $B$ sites, shown in Fig. 1(b), labeled Mn3/Ti1, are octahedrally coordinated and occupied by $75 \% \mathrm{Mn}^{3+}$ and $25 \% \mathrm{Ti}^{4+}$ for the $x=1$ sample, $50 \% \mathrm{Mn}^{3+}$ and $50 \% \mathrm{Ti}^{4+}$ for the $x=2$ sample, and $25 \% \mathrm{Mn}^{2+}$ and $75 \% \mathrm{Ti}^{4+}$ for the $x=3$ sample. The two cations are disordered across the $B$ sites with an average oxidation state of between +3.25 and +3.5 . Published DC magnetometry measurements show that samples with $x=1$, $x=2$, and $x=3$ undergo a single magnetic phase transition at temperatures of $T_{c}=27,62$, and $40 \mathrm{~K}$, respectively [19]. Here, we perform a quantitative neutron-diffraction study and show that all compositions undergo a transition to a longrange ordered magnetic phase below $T_{c}$ in which the Mn ions adopt a collinear ferrimagnetic structure. Remarkably, these results demonstrate an unusual increase in the ordering temperature between the $x=1$ and $x=2$ samples, despite an increase in the amount of nonmagnetic $\mathrm{Ti}^{4+}$ on the $B$-site (a)
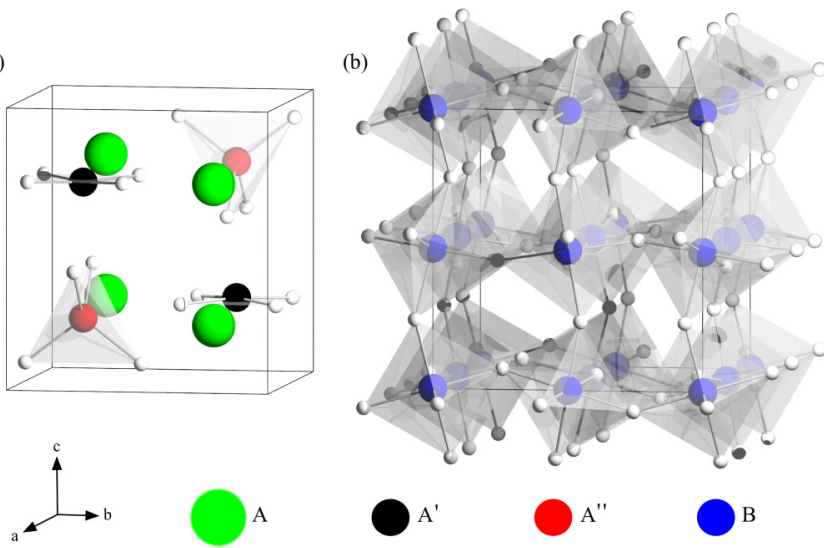

FIG. 1. Crystal structure of $\mathrm{Sm}_{2} \mathrm{MnMnMn}_{4-x} \mathrm{Ti}_{x} \mathrm{O}_{12}$ separated into (a) the $A$ sites and (b) the $B$ sites in the $P 4_{2} / n m c$ space group. The ions are represented by spheres with the $A$-site ions given in green, the $A^{\prime}$-site ions in black, the $A^{\prime \prime}$-site ions in red, the $B$-site ions in blue, and the $\mathrm{O}^{2-}$ anions in white. The unit cell is drawn in black, and $\mathrm{BO}_{6}$ octahedral coordinations are shaded in gray.

sublattice, which is contrary to what is observed in numerous other magnetically dilute systems [20] and is suggestive of a departure from mean-field physics. In the $x=2$ sample we demonstrate that site-selective magnetic dilution has enabled the tuning of ferrimagnetic compensation and the introduction of temperature-induced magnetization reversal. We show that in the $x=3$ sample, long-range $B$-site magnetic order must percolate via both $A-B$ and $B-B$ exchange, demonstrating the importance of $A-B$ exchange in mediating magnetism in the $A$-site columnar ordered quadruple perovskites.

The paper is organized as follows. In Sec. II we present the experimental details, followed by the results of the neutron powder-diffraction refinement, crystal electric field and magnetic anisotropy calculations, and percolation calculations in Secs. III A, III B, and III C, respectively. A discussion of the variation of ordering temperature and size of the magnetic moments with $\mathrm{Ti}^{4+}$ content is given in Sec. IV, and a summary of our findings is given in Sec. V.

\section{EXPERIMENTAL DETAILS}

Polycrystalline samples of $\mathrm{Sm}_{2} \mathrm{MnMnMn}_{4-x} \mathrm{Ti}_{x} \mathrm{O}_{12}$ were synthesized under high-temperature and high-pressure conditions from stoichiometric mixtures of $\mathrm{Mn}_{2} \mathrm{O}_{3}, \mathrm{Mn}_{3} \mathrm{O}_{4}, \mathrm{TiO}_{2}$, and $\mathrm{Sm}_{2} \mathrm{O}_{3}$ for $x=1, \mathrm{Mn}_{2} \mathrm{O}_{3}, \mathrm{MnO}, \mathrm{TiO}_{2}$, and $\mathrm{Sm}_{2} \mathrm{O}_{3}$ for $x=2$, and $\mathrm{MnO}, \mathrm{TiO}_{2}$, and $\mathrm{Sm}_{2} \mathrm{O}_{3}$ for $x=3$, as detailed in Ref. [19]. Neutron powder-diffraction experiments were performed using the time-of-flight diffractometer WISH at ISIS [21]. $x=1, x=2$, and $x=3$ samples of mass 0.8, 0.9, and $0.7 \mathrm{~g}$, respectively, were loaded into 3 -mm vanadium cans and cooled to $1.5 \mathrm{~K}$. Diffraction data with good counting statistics were collected on warming from a base temperature of $1.5 \mathrm{~K}$ up to $35 \mathrm{~K}$ in $5-\mathrm{K}$ steps for the $x=1$ sample, up to $78 \mathrm{~K}$ in $6-\mathrm{K}$ steps for the $x=2$ sample, and up to $60 \mathrm{~K}$ in $6-\mathrm{K}$ steps for the $x=3$ sample. Measurements with high counting statistics were made in the paramagnetic phase ( $80 \mathrm{~K}$ for $x=1$ and $x=3,90 \mathrm{~K}$ for $x=2$ ) and at $1.5 \mathrm{~K}$ for all three samples. Symmetry analysis was performed using 


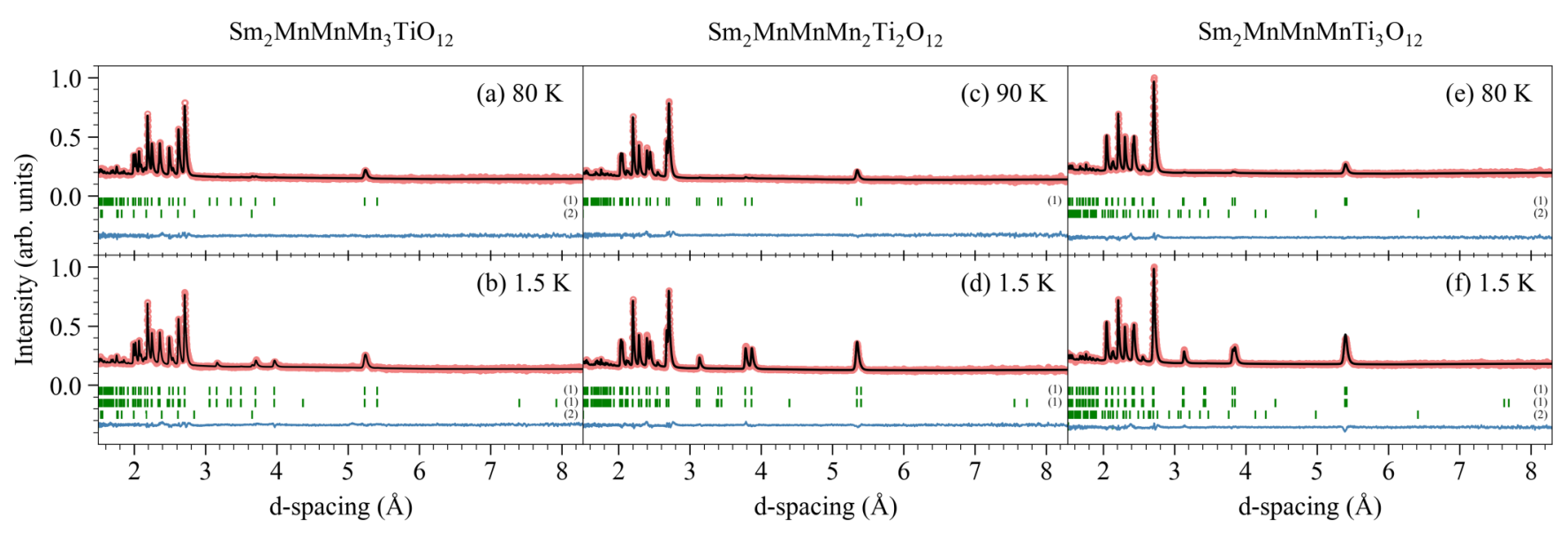

FIG. 2. Neutron powder-diffraction data collected on the time-of-flight diffractometer WISH at ISIS of (a) and (b) the $x=1$ sample, (c) and (d) the $x=2$ sample, and (e) and (f) the $x=3$ sample, at temperatures representative of the paramagnetic phase and at $1.5 \mathrm{~K}$. The data are given by the red circles, the fit of the data by the solid black line, and their difference as a blue line. The green tick marks are the nuclear and magnetic reflections from top to bottom of $\mathrm{Sm}_{2} \mathrm{MnMnMn}_{4-x} \mathrm{Ti}_{x} \mathrm{O}_{12}$, labeled "(1)," and an impurity phase, labeled "(2)."

the ISOTROPY software suite [22], and the diffraction data were fit using FULLPROF [23]. Magnetic measurements were performed on a superconducting quantum interference device (SQUID) magnetometer (Quantum Design, MPMS-XL-7T) between 2 and $400 \mathrm{~K}$ in 100 Oe under both zero-field-cooled (ZFC) and field-cooled-on-cooling (FCC) conditions. Isothermal magnetization measurements were performed between -70 and $70 \mathrm{kOe}$ at $5 \mathrm{~K}$.

\section{RESULTS}

\section{A. Crystal and magnetic structures}

The $P 4_{2} / n m c$ crystal structure model previously reported for $\mathrm{Sm}_{2} \mathrm{MnMnMn}_{4-x} \mathrm{Ti}_{x} \mathrm{O}_{12}$ [19] was refined against the neutron powder-diffraction data measured in the paramagnetic phase of each of the samples. For the $x=1$ and $x=3$ samples, weak diffraction peaks that could not be indexed by the $P 4_{2} / n m c$ model were found to originate in an $\mathrm{MnCO}_{3}$ impurity phase $(0.11$ wt $\%)$ and $\mathrm{Sm}_{2} \mathrm{Ti}_{2} \mathrm{O}_{7}$ impurity phase (0.25 wt \%), respectively. For all three samples the thermal parameter of Mn1 refined to unphysically large values when the ion was constrained to the center of the square-planar coordination (Wyckoff position 2a). Hence we adopted a split-atom model representative of site disorder above and below the square-planar coordination (Wyckoff position 4c), as employed in Ref. [19], which reduced the thermal parameter to physical values. Sm was free to substitute $\mathrm{Mn}$ on the $A^{\prime}$ and $A^{\prime \prime}$ sites, and $\mathrm{Mn}$ was free to substitute $\mathrm{Sm}$ on the $A$ sites, while imposing full site occupations. An excellent fit was achieved in all samples as shown in Fig. 2(a), 2(c) and 2(e), and the refined stoichiometries were as follows: $\mathrm{Sm}_{2} \mathrm{Mn}\left(\mathrm{Mn}_{0.84} \mathrm{Sm}_{0.16}\right) \mathrm{Mn}_{3} \mathrm{TiO}_{12}(R=2.72 \%, w R=2.90 \%$, $R_{\text {Bragg }}=4.06 \%$ at $\left.80 \mathrm{~K}\right), \mathrm{Sm}_{2} \mathrm{Mn}\left(\mathrm{Mn}_{0.93} \mathrm{Sm}_{0.07}\right) \mathrm{Mn}_{2} \mathrm{Ti}_{2} \mathrm{O}_{12}$ $\left(R=2.71 \%, w R=2.87 \%, R_{\mathrm{Bragg}}=5.05 \%\right.$ at $\left.90 \mathrm{~K}\right)$, and $\mathrm{Sm}_{2} \mathrm{MnMnMnTi}_{3} \mathrm{O}_{12}\left(R=2.03 \%, w R=2.20 \%, R_{\mathrm{Bragg}}=\right.$ $5.69 \%$ at $80 \mathrm{~K}$ ). Note that the $R_{\text {Bragg values are large in all }}$ samples due to strong absorption from $\mathrm{Sm}^{3+}$, which results in much weaker diffracted intensities.
A gradual growth of the (200), (002), (112), and (110) reflections was observed for all three samples below $T_{c}$ in accordance with changes in the sample's magnetization (Fig. 5), and hence these peaks were identified as magnetic in origin. For all three samples, the magnetic peaks are sharp and of a similar width to the nuclear peaks as shown in Fig. 2(b), 2(d) and 2(f), indicating that the magnetic structure is well correlated. The above peak positions were consistent with a $\Gamma$-point, $\mathbf{k}=(0,0,0)$, magnetic propagation vector. The magnetic $\Gamma$-point representation for the Wyckoff positions of the Sm and Mn sublattices decomposes into six 1-dimensional irreducible representations (irreps) $\left(\Gamma_{1}^{ \pm}, \Gamma_{2}^{ \pm}, \Gamma_{3}^{+}\right.$, and $\left.\Gamma_{4}^{+}\right)$and two 2-dimensional (2D) irreps $\left(\Gamma_{5}^{ \pm}\right)$, which together define a total of 12 different magnetic symmetries. The rapid increase in the magnetic susceptibility below $T_{c}$ (Fig. 5) indicated the presence of ferromagnetic sublattices, which are only consistent with the $\Gamma_{3}^{+}$or $\Gamma_{5}^{+}$irreps. Furthermore, the observation of finite magnetic intensity on the (002) reflection indicates that the magnetic moments are not aligned parallel to the $c$ axis (as magnetic diffraction intensity is proportional to the magnetic-moment components perpendicular to the scattering vector), which uniquely identifies that the magnetic structure transforms by the $\Gamma_{5}^{+}$irrep.

The $\Gamma_{5}^{+}$symmetry-adapted basis functions for the $A, A^{\prime}$, $A^{\prime \prime}$, and $B$ sites are summarized in Tables III, IV, and V of Appendix A. Magnetic structure models constructed from linear combinations of these basis functions were exhaustively tested by refinement against the neutron-diffraction data measured at $1.5 \mathrm{~K}$ from all samples. A model in which the $B$-site moments were oriented collinearly in the $a b$ plane and ferromagnetically coupled, and the $A^{\prime}$ - and $A^{\prime \prime}$-site moments were collinear but antialigned with respect to the $B$-site sublattice gave the best fit to the data $\left(R_{\mathrm{Mag}}=17.0 \%\right.$ for $x=1,5.39 \%$ for $x=2$, and $4.34 \%$ for $x=3$ ) and is shown in Fig. 3(a). The $A$-site $\mathrm{Sm}^{3+}$ moments refined to values lower than the sensitivity of the measurement $\left(\sim 0.1 \mu_{\mathrm{B}}\right)$; hence they were fixed to zero. The refined moment magnitudes are given in Table I, which also lists the net ferrimagnetic moment per formula unit. The strong dependence of the net ferrimagnetic 


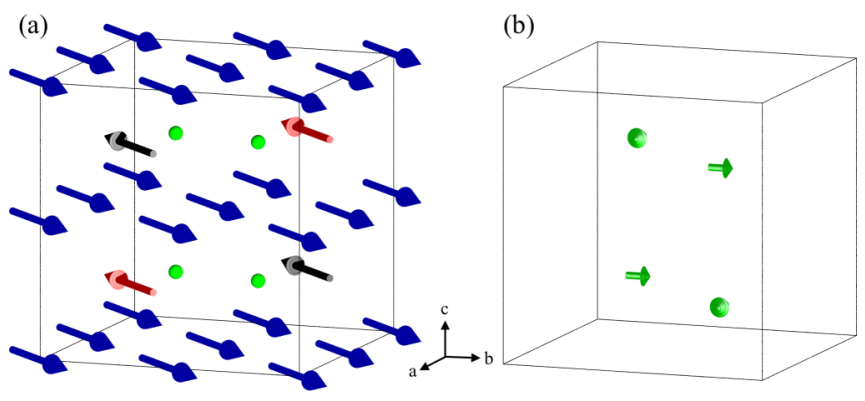

FIG. 3. (a) Experimentally determined magnetic structure of $\mathrm{Sm}_{2} \mathrm{MnMnMn}_{4-x} \mathrm{Ti}_{x} \mathrm{O}_{12}$ up to a direction in the $a b$ plane. (b) Theoretically proposed spin configuration of the $\mathrm{Sm} 1$ sites as determined by point-charge calculations. The $A$ sites are colored in green, the $A^{\prime}$ sites in black, and the $A^{\prime \prime}$ sites in red. The $B$ sites are colored in blue, and for clarity we show the scenario in which all $B$-site ions of a magnetic unit cell are Mn ions (a more accurate depiction of the unit cell would consist of statistically disordered $\mathrm{Ti}^{4+}$ and $\mathrm{Mn}$ ions on the $B$ sites). The magnetic space group is $C c c^{\prime} a^{\prime}$ (No. 68.516) with basis vectors $(1,1,0),(-1,1,0),(0,0,1)$ and an origin of $(1,0,1 / 2)$ with respect to the paramagnetic space group.

moment on composition $x$ is reflected in the remnant magnetization $M_{r}$, evaluated by extrapolating the powder-averaged bulk magnetization to zero field (see Fig. 4). Scaled extrapolated values are listed in Table I, and their relative magnitudes correspond well to the net ferrimagnetic moment per formula unit obtained from neutron diffraction, except for the $x=2$ sample. This discrepancy cannot be explained by the proposed magnetic structure model.

The refined magnetic structure is consistent with the collinear ferrimagnetic phase found in $\mathrm{Tm}_{2} \mathrm{MnMnMn}_{4} \mathrm{O}_{12}$ [17] and $R_{2} \mathrm{CuMnMn}_{4} \mathrm{O}_{12}$ [18]. Moment orientations of $m\|[x, 0,0], m\|[x, x, 0]$, or $m \|[x, y, 0]$ correspond to different magnetic symmetries described by the three distinct order-parameter directions of the $\Gamma_{5}^{+}$irrep (see Tables III, IV, and V of Appendix A); however, these three

TABLE I. Absolute values of the 1.5-K experimental (Expt.) and theoretical (Calc.) ionic magnetic moments of $\mathrm{Sm}_{2} \mathrm{MnMnMn}_{4-x} \mathrm{Ti}_{x} \mathrm{O}_{12}$. The net magnetization $M$ per formula unit is determined from the experimental moment values, where the $A$ sites align antiparallel to the $B$ sites. Note that the refined Sm1 moment was fixed to zero. The remnant magnetization $M_{r}$ extrapolated from magnetometry data was scaled by a factor of 2 to account for powder averaging. All moments are given in units of $\mu_{\mathrm{B}}$.

\begin{tabular}{|c|c|c|c|c|c|c|}
\hline \multirow[b]{2}{*}{ Ion } & \multicolumn{2}{|c|}{$x=1$} & \multicolumn{2}{|c|}{$x=2$} & \multicolumn{2}{|c|}{$x=3$} \\
\hline & Expt. & Calc. & Expt. & Calc. & Expt. & Calc. \\
\hline Sm1 & 0.00 & 0.71 & 0.00 & 0.71 & 0.00 & 0.71 \\
\hline Mn1 & $1.70(7)$ & 4.0 & $3.72(7)$ & 5.0 & $4.09(8)$ & 5.0 \\
\hline Mn2 & $2.03(9)$ & 5.0 & $3.72(7)$ & 5.0 & $4.09(8)$ & 5.0 \\
\hline Mn3 & $0.82(6)$ & 4.0 & $2.54(6)$ & 4.0 & 2.1(1) & 5.0 \\
\hline$M$ per f.u. & \multicolumn{2}{|c|}{$1.3(1)$} & \multicolumn{2}{|c|}{$2.4(1)$} & \multicolumn{2}{|c|}{$6.0(2)$} \\
\hline$M_{r}$ per f.u. & \multicolumn{2}{|c|}{$1.006(7)$} & \multicolumn{2}{|c|}{$1.05(1)$} & \multicolumn{2}{|c|}{$5.68(3)$} \\
\hline$T_{c}(\mathrm{~K})$ & \multicolumn{2}{|c|}{27} & \multicolumn{2}{|c|}{62} & \multicolumn{2}{|c|}{34} \\
\hline
\end{tabular}

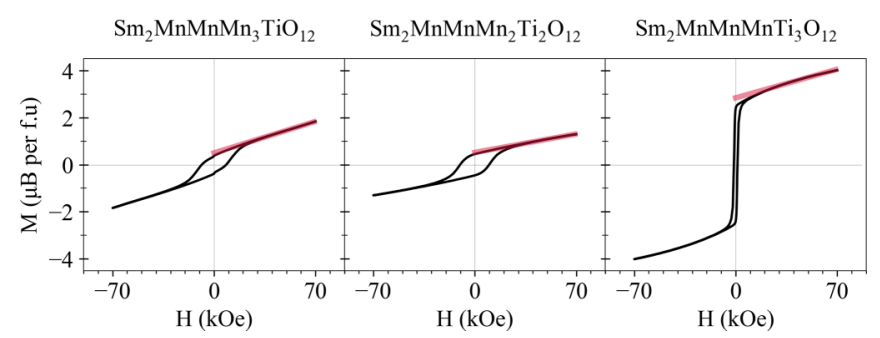

FIG. 4. Plots of the magnetization $M$ as a function of applied field $H$ taken at a temperature of $5 \mathrm{~K}$ for each of the samples. A magnetic field range of $22.5-70 \mathrm{kOe}$ was used to extrapolate the magnetization to zero field (the red line).

cases cannot be differentiated by our neutron-diffraction data due to powder averaging in the tetragonal crystal symmetry. In the following we adopt a model with $m \|[x, x, 0]$, which is consistent with $\mathrm{Sm}^{3+}$ crystal electric field calculations (Sec. III B).

The temperature dependences of all symmetryinequivalent moment magnitudes, plotted in Fig. 5, were evaluated by fitting the magnetic structure model to variable-temperature neutron powder-diffraction data measured from all three samples. The refinements were constrained such that each magnetic ion had a moment no larger than its theoretically predicted maximum, given in Table I, and with the ratio of the Mn1:Mn2 moments chosen to be consistent with their nominal oxidation states. The refinement was insensitive to a Sm1 moment at all temperatures, so it was set to zero. For the $x=2$ sample the $B$-site moments grow rapidly as the temperature decreases but quickly saturate in comparison to the $A$-site moments, which steadily grow with decreasing temperature. As a result, the net magnetization (the sum over all magnetic moments in the unit cell with the $A$ sublattices antialigned with respect to the $B$ sublattice) reverses direction with decreasing temperature, giving rise to a compensation point at $45 \pm 3 \mathrm{~K}$. Magnetization reversal was also observed in the temperature-dependent magnetic susceptibility FCC data of the $x=2$ sample, as shown in the inset of Fig. 5(e), for which the compensation temperature was determined to be $46 \pm 1 \mathrm{~K}$, in excellent agreement with the neutron-diffraction results. We note that the absolute value of the negative, low-temperature magnetic susceptibility is not reflective of the full net ferrimagnetic moment, as the coercive field of the sample may come close to the 100-Oe applied field.

\section{B. Magnetic anisotropy}

The Jahn-Teller active $\mathrm{Mn}^{3+}$ ions present in the $x=1$ and $x=2$ samples are expected to impose an average weak single-ion anisotropy $\| c[17,24]$, while all other Mn ions are isotropic $\mathrm{Mn}^{2+}$. The three $\mathrm{Sm}_{2} \mathrm{MnMnMn}_{4-x} \mathrm{Ti}_{x} \mathrm{O}_{12}$ samples have a magnetization direction in the $a b$ plane, perpendicular to the easy axis of the $\mathrm{Mn}^{3+}$ ions, suggesting that the magnetic anisotropy of $\mathrm{Sm}^{3+}$ may play an important role despite our data being insensitive to an ordered moment on the Sm1 sites. We note that in $\mathrm{Tm}_{2} \mathrm{MnMnMn}_{4} \mathrm{O}_{12}$ the competition between the $\mathrm{Mn}^{3+}$ and rare-earth (RE) magnetic anisotropies was found to result in a spin-reorientation phase transition. 


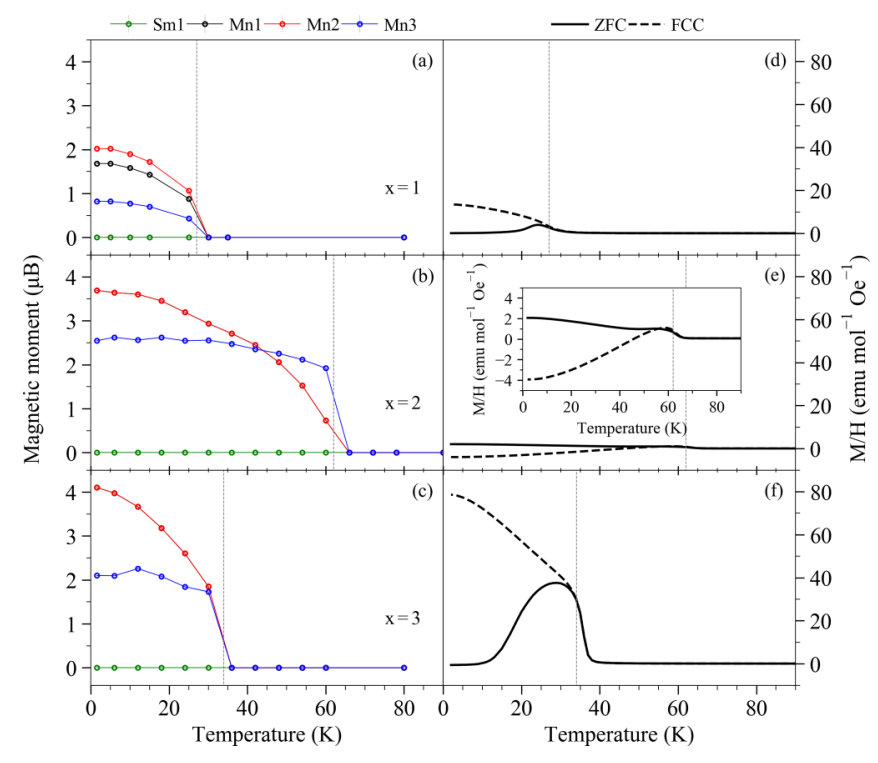

FIG. 5. (a)-(c) The temperature dependence of ionic moment magnitudes given for each of the symmetry-inequivalent magnetic ions. The moments of $\mathrm{Mn} 1$ and $\mathrm{Mn} 2$ were fixed to be the same in the $x=2$ and $x=3$ samples to be consistent with their equivalent nominal oxidation states. (d)-(f) ZFC and FCC magnetization measurements under an applied DC field of 100 Oe. The inset in (e) shows the ZFC and FCC measurements for the $x=2$ sample on a reduced scale.

If the magnetic anisotropy is indeed determined by $\mathrm{Sm}^{3+}$, then the absence of a spin-reorientation phase transition in the $\mathrm{Sm}_{2} \mathrm{MnMnMn}_{4-x} \mathrm{Ti}_{x} \mathrm{O}_{12}$ compounds implies significant $f$-d exchange coupling immediately below $T_{c}$ (note that in the orthoferrites, strong $f$ - $d$ exchange coupling is also present in $\mathrm{SmFeO}_{3}$ and leads to a phase transition approximately 300-400 K above all other rare-earth orthoferrites [25]).

Point-charge approximations to the $\mathrm{Sm}^{3+}$ crystal electric field (CEF) were used to calculate the ground-state magnetic anisotropy of $\mathrm{Sm}^{3+}$ and hence establish whether $f-d$ exchange coupling of rare-earth moments to the manganese sublattices might be responsible for the $a b$-plane magnetization direction observed in $\mathrm{Sm}_{2} \mathrm{MnMnMn}_{4-x} \mathrm{Ti}_{x} \mathrm{O}_{12} . \mathrm{Sm}^{3+}$ has electronic configuration [Xe $] 4 f^{5}$, and Hund's rules give $S=5 / 2, L=5$, and $J=5 / 2$ for the lowest-energy sixfold multiplet of states. The local crystal electric field of $\mathrm{Sm}^{3+}$ has the point-group symmetry $2 \mathrm{~mm}$, which lifts the multiplet degeneracy to form three Kramers doublets. All nonzero crystal electric field parameters, $B_{n}^{m}$ [26], were calculated for $\mathrm{Sm}^{3+}$ in its two ionic positions, $\left(\frac{1}{4}, \frac{1}{4}, z\right)$ and $\left(\frac{1}{4}, \frac{1}{4}, z+\frac{1}{2}\right)$, labeled Sm11 and Sm12, respectively (Table II). Ionic positions refined for the $x=2$ compound at $1.5 \mathrm{~K}$ were used for

TABLE II. Nonzero crystal electric field parameters for $\mathrm{Sm}^{3+}$ in $\mathrm{Sm}_{2} \mathrm{MnMnMn}_{2} \mathrm{Ti}_{2} \mathrm{O}_{12}$ evaluated by the point-charge model and given in units of $\mu \mathrm{eV}$.

\begin{tabular}{lcccccc}
\hline \hline Ion & Fractional coordinates & $B_{2}^{0}$ & $B_{2}^{2}$ & $B_{4}^{0}$ & $B_{4}^{2}$ & $B_{4}^{4}$ \\
\hline Sm11 & $0.25,0.25,0.209$ & 2430 & 5204 & -11.65 & 40.94 & 41.85 \\
Sm12 & $0.25,0.25,0.709$ & 2430 & -5204 & -11.65 & -40.94 & 41.85 \\
\hline \hline
\end{tabular}

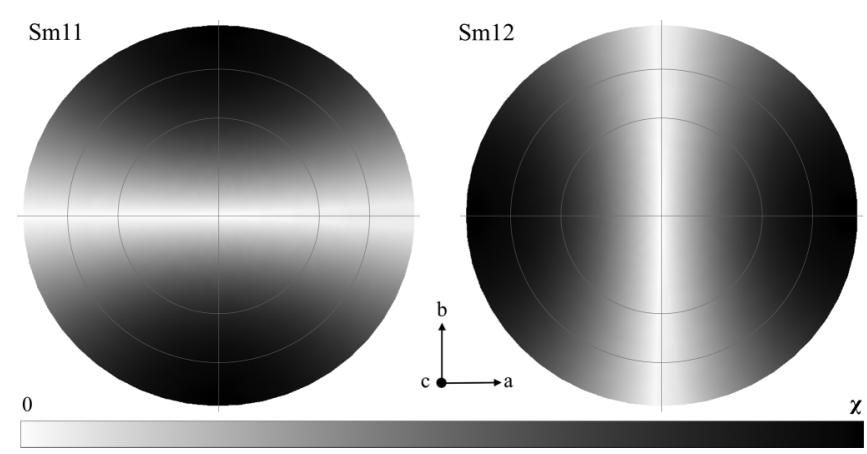

FIG. 6. The magnetic susceptibility of Sm11 and Sm12 plotted as a stereographic projection over a full hemisphere under an applied field of $1 \mathrm{~T}$.

reference, and similar values were obtained using the ionic positions of the $x=1$ and $x=3$ samples. Sm11 and Sm12 are symmetry equivalent and related by a $4_{2}$ screw; therefore all values of $B_{n}^{m}$ are the same across the two sites, except the $B_{2}^{2}$ and $B_{4}^{2}$ terms, which change sign upon $90^{\circ}$ rotation about $c$.

We consider the following Hamiltonian for a single $\mathrm{Sm}^{3+}$ ion:

$$
\mathcal{H}=\sum_{n} \sum_{m=-n}^{n} B_{n}^{m} O_{n}^{m}+g_{J} \mu_{\mathrm{B}} \mathbf{J} \cdot \mathbf{B}_{\mathrm{ex}},
$$

where $O_{n}^{m}$ are the Stevens operators [26], $g_{J}$ is the Landé $g$ factor, $\mathbf{J}=\left(J_{x}, J_{y}, J_{z}\right)$ is the vector total angular momentum operator, and $\mathbf{B}_{\mathrm{ex}}$ is an effective exchange field originating in $f$ - $d$ exchange interactions with the manganese sublattices.

The magnetic anisotropy of Sm11 and Sm12 was determined by repeatedly solving Eq. (1) for a 1-T field applied in different directions over a full hemisphere. Eigenfunctions were found through diagonalization of $\mathcal{H}$, and magneticmoment components $m_{x}, m_{y}$, and $m_{z}$ were calculated by summing the expectation values of $\left\langle J_{x}\right\rangle,\left\langle J_{y}\right\rangle$, and $\left\langle J_{z}\right\rangle$ over all eigenfunctions, multiplied by $g_{J}$ and weighted by Boltzmann statistics evaluated at $T=10 \mathrm{~K}$.

The total Sm11 and Sm12 moments for the different field directions are shown in Fig. 6 as a stereographic projection. A strong Ising-like anisotropy was found $\| y$ for Sm11 and $\| x$ for $\mathrm{Sm} 12$. The easy axis rotates between $x$ and $y$ for $\mathrm{Sm}^{3+}$ sites in accordance with the $4_{2}$ screw symmetry that relates the two sites. We note that this perpendicular alignment of Sm11 and Sm12 moments parallel to $y$ and $x$, respectively, is described by the B1 symmetry-adapted mode associated with the $(a, a)$ order-parameter direction of $\Gamma_{5}^{+}$(see Table III of Appendix A). Within this symmetry, the Sm moments only couple to the Mn collinear ferrimagnetic structure if the Mn moments lie $\|[x, x, 0]$. Hence not only can the $\mathrm{Sm}^{3+}$ ions impose an $a b$-plane magnetic anisotropy, but also they can determine the direction of the magnetization in the plane [as shown in Fig. 3(b)].

\section{Percolation calculations}

First, we consider the percolation of long-range magnetic order via $B-B$ exchange alone. In $\mathrm{Sm}_{2} \mathrm{MnMnMn}_{4-x} \mathrm{Ti}_{x} \mathrm{O}_{12}$, 

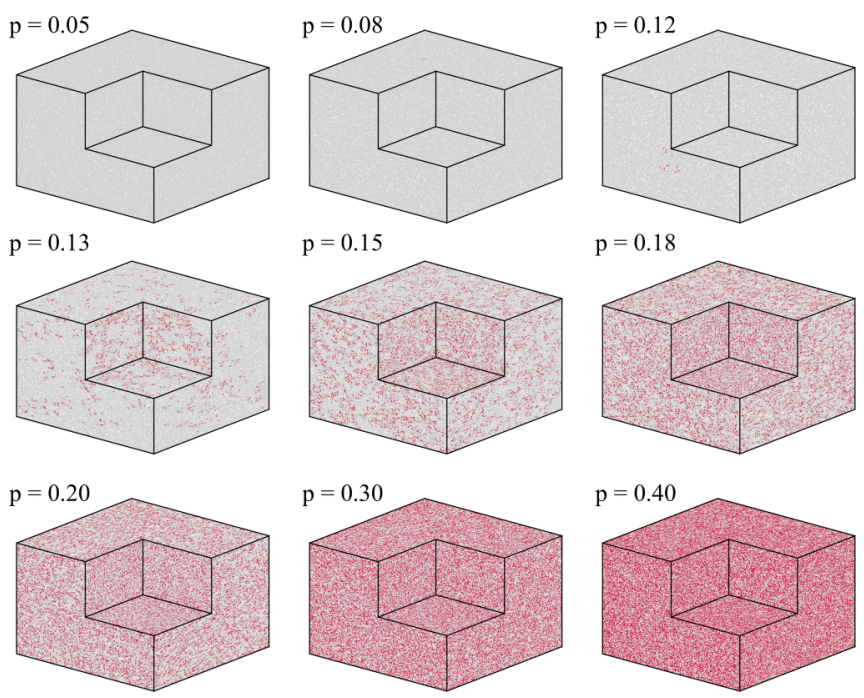

FIG. 7. A three-dimensional illustration of a cubic lattice, spanning $100 A_{2} A^{\prime} A^{\prime \prime} B_{4} \mathrm{O}_{12}$ unit cells in each of the three Cartesian directions and showing only clusters of $B$-site ions when magnetic order is percolated by both $A-B$ and $B-B$ exchange, given for nine different values of the lattice occupation $p$. In each figure, only voxels that intersect the surface are shown, and an eighth of the cubic lattice has been removed to reveal the interior. The largest Mn cluster is given in red; all other sites occupied by $\mathrm{Mn}$ are given in white, and sites occupied by nonmagnetic $\mathrm{Ti}^{4+}$ are in gray.

$B-B$ exchange pathways span a three-dimensional framework. Each $B$ site has six nearest-neighbor $B$ sites, such that for every nonmagnetic $\mathrm{Ti}^{4+}$ ion substituted onto this sublattice, six nearest-neighbor magnetic $B-B$ exchange pathways are removed. The site-percolation threshold (the probablility $p$ of $\mathrm{Mn} B$-site occupation required to establish a percolating cluster that spans the entire lattice between two opposing faces) for a simple cubic lattice representative of the $B-B$ framework is $31.16 \%$ [27]. Hence $B-B$ exchange is expected to play no role in establishing long-range magnetic order in the $x=3$ sample, for which the $B$-site Mn occupation is $25 \%$.

To explore the origin of long-range magnetic order in the $x=3$ sample, we calculated the percolation threshold if magnetic order were to percolate via both $A-B$ and $B-B$ exchange. Two interpenetrating simple cubic lattices were used to represent the $A_{2} A^{\prime} A^{\prime \prime} B_{4} \mathrm{O}_{12}$ unit cell, where the $\mathrm{Mn}$ $A^{\prime}$ and $A^{\prime \prime}$ sites were set to be fully occupied and the $\operatorname{Sm} A$ sites were set to be unoccupied to exclude interactions with the RE ions. Mn ions were randomly placed on the $B$ sites according to a probability of occupation $p$. For a given $p$, the sites were sorted into clusters, where a cluster is composed of a continuous network of nearest-neighbor $\mathrm{Mn}$ ions, separated from other clusters by $\mathrm{Ti}^{4+}$ ions. The percolation threshold was evaluated as the minimum value of $p$ for which there exists a cluster that percolates through the entire lattice along the $c$ axis.

We found that in this case the percolation threshold was reduced from 31.16 to $12.4(1) \%$, as shown in Fig. 7, demonstrating that $A-B$ exchange is crucial to mediating long-range magnetic order in the $x=3$ sample.

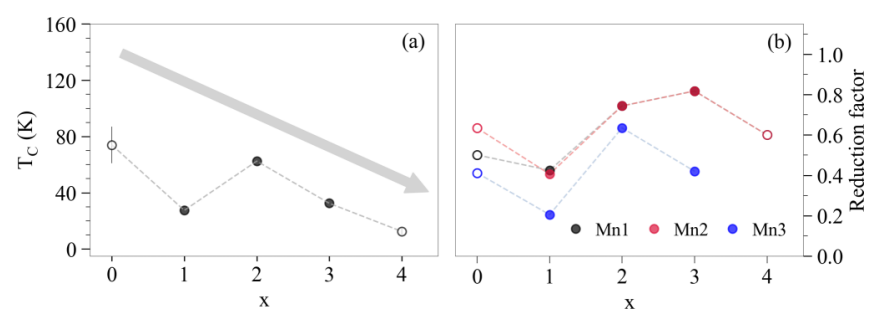

FIG. 8. The dependence of (a) the ordering temperature $T_{c}$ and (b) the reduction in magnetic moment relative to the full $g\left\langle J_{z}\right\rangle \mu_{\mathrm{B}}$ ground-state value on the concentration of nonmagnetic $\mathrm{Ti}^{4+}$ (labeled by $x$ ). The data for the $x=0$ and $x=4$ samples are from $\mathrm{Tm}_{2} \mathrm{MnMnMn}_{4} \mathrm{O}_{12}$ [17] and $(\mathrm{NaDy}) \mathrm{MnMnTi}_{4} \mathrm{O}_{12}$ [16], where the vertical bars indicate the range of known transition temperatures for the $R_{2} \mathrm{MnMnMn}_{4} \mathrm{O}_{12}$ [15] and $(\mathrm{NaR}) \mathrm{MnMnTi}_{4} \mathrm{O}_{12}$ [16] samples, respectively. The gray arrow in (a) is used to illustrate the trend of the mean-field dependence of the ordering temperature with $x$.

We note that $A-A$ exchange could also percolate long-range magnetic order; however, measurements of $(\mathrm{NaDy}) \mathrm{MnMnTi}_{4} \mathrm{O}_{12}$ demonstrated that it does so at temperatures of $12 \mathrm{~K}$ [16], which is significantly below the ordering temperatures of all measured $\mathrm{Sm}_{2} \mathrm{MnMnMn}_{4-x} \mathrm{Ti}_{x} \mathrm{O}_{12}$ samples.

\section{DISCUSSION}

Diluting a magnetic sublattice with nonmagnetic ions is predicted to decrease the magnetic ordering temperature $[20,28]$, and at the mean-field level one expects an approximately linear dependence in the transition temperature on magnetic ion concentration [29-31]. Remarkably, the ordering temperature of the $\mathrm{Sm}_{2} \mathrm{MnMnMn}_{4-x} \mathrm{Ti}_{x} \mathrm{O}_{12}$ solid solution does not smoothly decrease upon increasing $x$ [Fig. 8(a)], which indicates a significant departure from mean-field physics in this system [32] that is also reflected in the moment magnitudes. In insulating materials the magnetic moment of a given ion should reach its full $g\left\langle J_{z}\right\rangle \mu_{\mathrm{B}}$ value in the ground state. The ground-state magnetic moments refined for $\mathrm{Sm}_{2} \mathrm{MnMnMn}_{4-x} \mathrm{Ti}_{x} \mathrm{O}_{12}$ are compared with their theoretical values in Table I and plotted in terms of a relative reduction factor in Fig. 8(b). A considerable departure from the full moment is observed for all $\mathrm{Sm}_{2} \mathrm{MnMnMn}_{4-x} \mathrm{Ti}_{x} \mathrm{O}_{12}$ samples, and the relative reduction in moment magnitude [Fig. 8(b)] correlates well with the nonlinear variation observed in $T_{\mathrm{c}}$ [Fig. 8(a)]. In diffraction the time-averaged correlated moment is measured, and therefore a reduction in the ground-state moment magnitudes could originate from strong spin fluctuations and/or static disorder.

Ideally, we would be able to perform the same analysis on an $x=0$ sample, which has a fully magnetic $B$-site sublattice, and an $x=4$ sample, which has a nonmagnetic $B$-site sublattice. However, the former can only be grown in very small quantities [15], and the latter requires the $A$-site Mn ions to adopt an unfeasible +1 oxidation state. Instead, we have used the moment magnitudes and ordering temperature for $\mathrm{Tm}_{2} \mathrm{MnMnMn}_{4} \mathrm{O}_{12}$, as indicative of the $x=0$ sample, and the ordering temperature and moment magnitude of ( $\mathrm{NaDy}) \mathrm{MnMnTi}_{4} \mathrm{O}_{12}$, as indicative of the $x=4$ sample, 
plotted in Fig. 8 as open circles. Importantly, these data further support the correlation between the reduction in the relative moment magnitudes and the ordering temperature-an effect that appears to be prevalent in the broader family of materials.

In $\mathrm{Sm}_{2} \mathrm{MnMnMn}_{4-x} \mathrm{Ti}_{x} \mathrm{O}_{12}$, static disorder of the magnetic structure may originate in at least one of two forms of crystallographic disorder inherent to these compounds: (i) mixed cation occupation and (ii) displacements of Mn1 ions above and below their coordination plane. All nuclear neutron-diffraction peaks were found to be sharp and limited by the instrumental resolution, indicating that either form of crystallographic disorder statistically effected every unit cell equally, giving a crystal structure that is well correlated on average. The magnetic diffraction peaks had widths similar to those of the nuclear peaks, indicating that the magnetic structures were also well correlated on average, such that any static disorder of the magnetic structure must be statistical in nature (i.e., extensive inhomogeneities are not prevalent). Static disorder is therefore unlikely to lead to a departure from mean-field behavior.

By comparison, spin fluctuations can coexist with wellcorrelated magnetic order [33], have been shown to reduce moment magnitudes even at low temperature [34], and could naturally gives rise to non-mean-field behavior that may be prevalent in the $x=1$ and $x=3$ samples, and weakened in the $x=2$ sample. The prevalence of spin fluctuations is emerging in the wider family of $A$-site columnar ordered quadruple perovskites that support competing exchange pathways. For example, in $R_{2} \mathrm{CuMnMn}_{4} \mathrm{O}_{12}\left(R=\mathrm{Y}\right.$ or Dy), $B$-site $\mathrm{Mn}^{3+}$ moments appear to saturate at $\frac{3}{4}$ of their theoretical value, and only on cooling through a low-temperature phase transition, proposed to originate in the softening of low-energy magnons, do they recover their full moment [18].

\section{CONCLUSIONS}

All three magnetically dilute $x=1, x=2$, and $x=3$ $\mathrm{Sm}_{2} \mathrm{MnMnMn}_{4-x} \mathrm{Ti}_{x} \mathrm{O}_{12}$ samples adopt long-range magnetically ordered phases below 27,62 , and $34 \mathrm{~K}$, respectively. The $\mathrm{Mn}$ ions were empirically found to adopt a collinear ferrimagnetic structure up to a direction in the $a b$ plane, and we propose that the moment direction within the plane is likely determined by $f$ - $d$ exchange interactions between the $\mathrm{Mn}$ and Sm sublattices. We show that the introduction of 50\% magnetic dilution onto the $B$-site sublattice gives rise to a ferrimagnetic compensation point and magnetization reversal. Percolation calculations demonstrated that long-range magnetic order in the $x=3$ sample can only occur if it percolates via both $A-B$ and $B-B$ exchange, hence demonstrating the importance of $A-B$ exchange in the $A$-site columnar ordered quadruple perovskite manganites. Finally, we showed that the unusual variation in transition temperature that occurred upon magnetic dilution was reflected in the reduction of groundstate magnetic moments observed on all $\mathrm{Mn}$ sites and in all samples. Together, these results suggest the presence of spin fluctuations leading to a departure from mean-field physics. In future studies it would be interesting to perform inelastic neutron-scattering experiments to measure the $\mathrm{Sm}^{3+} \mathrm{CEF}$ energy levels and further refine our model of the magnetic anisotropy, as well as probe the presence of spin fluctuations at the lowest measured temperatures.

\section{ACKNOWLEDGMENTS}

R.D.J. acknowledges financial support from the Royal Society. K.Y. and A.A.B. acknowledge JSPS KAKENHI (Grant No. JP20H05276), the Nippon Sheet Glass Foundation for Materials Science and Engineering (Grant No. 40-37), and Innovative Science and Technology Initiative for Security from Acquisition, Technology, and Logistics Agency (ATLA), Japan (Grant No. JPJ004596).

\section{APPENDIX A: IRREDUCIBLE REPRESENTATIONS AND THEIR SYMMETRY-ADAPTED BASIS FUNCTIONS}

We give the basis functions of the $\Gamma_{5}^{+}$irreducible representation used to describe the magnetic structures of $\mathrm{Sm}_{2} \mathrm{MnMnMn}_{4-x} \mathrm{Ti}_{x} \mathrm{O}_{12}$ for the $A, A^{\prime}$ and $A^{\prime \prime}$, and $B$ sites in Tables III, IV, and V, respectively.

TABLE III. The $\Gamma_{5}^{+}$symmetry-adapted basis functions given for the $A$-site Wyckoff position in $\mathrm{Sm}_{2} \mathrm{MnMnMn}_{4-x} \mathrm{Ti}_{x} \mathrm{O}_{12}$, listed separately for the $(a, 0),(a, a)$, and $(a, b)$ order-parameter (O. P.) directions. Moment components of symmetry-equivalent ions have the same magnitude. Note that the modes and irreps are listed according to the labeling scheme adopted in the ISOTROPY software suite [22].

\begin{tabular}{|c|c|c|c|c|c|}
\hline O. P. & Mode & $0.25,0.25, z$ & $0.75,0.75,-z$ & $0.25,0.25, z+\frac{1}{2}$ & $0.75,0.75,-z+\frac{1}{2}$ \\
\hline \multirow[t]{2}{*}{$(a, 0)$} & $\mathrm{B} 2(a)$ & {$[1,0,0]$} & {$[1,0,0]$} & {$[0,0,0]$} & {$[0,0,0]$} \\
\hline & $\mathrm{B} 1(a)$ & {$[0,0,0]$} & {$[0,0,0]$} & {$[1,0,0]$} & {$[1,0,0]$} \\
\hline \multirow[t]{2}{*}{$(a, a)$} & $\mathrm{B} 2(a)$ & {$[1,0,0]$} & {$[1,0,0]$} & {$[0,1,0]$} & {$[0,1,0]$} \\
\hline & $\mathrm{B} 1(a)$ & {$[0,1,0]$} & {$[0,1,0]$} & {$[1,0,0]$} & {$[1,0,0]$} \\
\hline \multirow[t]{3}{*}{$(a, b)$} & $\mathrm{B} 2(a)$ & {$[1,0,0]$} & {$[1,0,0]$} & {$[0,0,0]$} & {$[0,0,0]$} \\
\hline & $\mathrm{B} 1(a)$ & {$[0,0,0]$} & {$[0,0,0]$} & {$[1,0,0]$} & {$[1,0,0]$} \\
\hline & $\mathrm{B} 1(b)$ & {$[0,1,0]$} & {$[0,1,0]$} & {$[0,0,0]$} & {$[0,0,0]$} \\
\hline
\end{tabular}


TABLE IV. The $\Gamma_{5}^{+}$symmetry-adapted basis functions given for the $A^{\prime}$ - and $A^{\prime \prime}$-site Wyckoff positions in $\mathrm{Sm}_{2} \mathrm{MnMnMn}_{4-x} \mathrm{Ti}_{x} \mathrm{O}_{12}$, listed separately for the $(a, 0),(a, a)$, and $(a, b)$ order-parameter directions. Here, we use Wyckoff position 2a of the $A^{\prime}$ site, such that it is constrained to the center of the square-planar coordination. Moment components of symmetry-equivalent ions have the same magnitude. Note that the modes and irreps are listed according to the labeling scheme adopted in the ISOTROPY software suite [22].

\begin{tabular}{lccc}
\hline \hline O. P. & Mode & $0.75,0.25,0.75$ & $0.25,0.75,0.25$ \\
\hline$(a, 0)$ & $\mathrm{E}(a)$ & {$[1,0,0]$} & {$[1,0,0]$} \\
$(a, a)$ & $\mathrm{E}(a)$ & {$[1,1,0]$} & {$[1,1,0]$} \\
$(a, b)$ & $\mathrm{E}(a)$ & {$[1,0,0]$} & {$[1,0,0]$} \\
& $\mathrm{E}(b)$ & {$[0,1,0]$} & {$[0,1,0]$} \\
\hline \hline
\end{tabular}

\section{APPENDIX B: CRYSTAL STRUCTURE PARAMETERS OF $\mathrm{Sm}_{2} \mathrm{MnMnMn}_{4-x} \mathrm{Ti}_{x} \mathrm{O}_{12}$}

We give the crystal structure parameters of $\mathrm{Sm}_{2} \mathrm{MnMnMn}_{4-x} \mathrm{Ti}_{x} \mathrm{O}_{12}$ for $x=1, x=2$, and $x=3$ in Tables VI, VII, and VIII, respectively.

TABLE V. The $\Gamma_{5}^{+}$symmetry-adapted basis functions given for the $B$-site Wyckoff position in $\operatorname{Sm}_{2} \mathrm{MnMnMn}_{4-x} \mathrm{Ti}_{x} \mathrm{O}_{12}$, listed separately for the $(a, 0),(a, a)$, and $(a, b)$ order-parameter directions. Moment components of symmetry-equivalent ions have the same magnitude. Note that the modes and irreps are listed according to the labeling scheme adopted in the ISOTROPY software suite [22].

\begin{tabular}{|c|c|c|c|c|c|c|c|c|c|}
\hline O. P. & Mode & $0.0,0.0,0.0$ & $0.5,0.0,0.0$ & $0.0,0.5,0.0$ & $0.5,0.5,0.0$ & $0.0,0.0,0.5$ & $0.5,0.0,0.5$ & $0.0,0.5,0.5$ & $0.5,0.5,0.5$ \\
\hline & $\operatorname{Ag}_{2}(a)$ & {$[0,0,0]$} & {$[0,0,0]$} & {$[0,0,0]$} & {$[0,0,0]$} & {$[0,1,0]$} & {$[0,-1,0]$} & {$[0,-1,0]$} & {$[0,1,0]$} \\
\hline & $\operatorname{Ag}_{4}(a)$ & {$[0,0,0]$} & {$[0,0,0]$} & {$[0,0,0]$} & {$[0,0,0]$} & {$[1,0,0]$} & {$[1,0,0]$} & {$[1,0,0]$} & {$[1,0,0]$} \\
\hline & $\operatorname{Ag}_{5}(a)$ & {$[0,0,1]$} & {$[0,0,-1]$} & {$[0,0,1]$} & {$[0,0,-1]$} & {$[0,0,0]$} & {$[0,0,0]$} & {$[0,0,0]$} & {$[0,0,0]$} \\
\hline \multirow{5}{*}{$(a, a)$} & $\operatorname{Ag}_{2}(a)$ & {$[1,0,0]$} & {$[-1,0,0]$} & {$[-1,0,0]$} & {$[1,0,0]$} & {$[0,1,0]$} & {$[0,-1,0]$} & {$[0,-1,0]$} & {$[0,1,0]$} \\
\hline & $\operatorname{Ag}_{3}(a)$ & {$[0,1,0]$} & {$[0,-1,0]$} & {$[0,-1,0]$} & {$[0,1,0]$} & {$[1,0,0]$} & {$[-1,0,0]$} & {$[-1,0,0]$} & {$[1,0,0]$} \\
\hline & $\operatorname{Ag}_{4}(a)$ & {$[0,1,0]$} & {$[0,1,0]$} & {$[0,1,0]$} & {$[0,1,0]$} & {$[1,0,0]$} & {$[1,0,0]$} & {$[1,0,0]$} & {$[1,0,0]$} \\
\hline & $\operatorname{Ag}_{5}(a)$ & {$[0,0,1]$} & {$[0,0,-1]$} & {$[0,0,1]$} & {$[0,0,-1]$} & {$[0,0,1]$} & {$[0,0,1]$} & {$[0,0,-1]$} & {$[0,0,-1]$} \\
\hline & $\operatorname{Ag}_{6}(a)$ & {$[0,0,1]$} & {$[0,0,1]$} & {$[0,0,-1]$} & {$[0,0,-1]$} & {$[0,0,1]$} & {$[0,0,-1]$} & {$[0,0,1]$} & {$[0,0,-1]$} \\
\hline & $\mathrm{Ag}_{2}(b)$ & {$[1,0,0]$} & {$[-1,0,0]$} & {$[-1,0,0]$} & {$[1,0,0]$} & {$[0,0,0]$} & {$[0,0,0]$} & {$[0,0,0]$} & {$[0,0,0]$} \\
\hline & $\operatorname{Ag}_{3}(a)$ & {$[0,1,0]$} & {$[0,-1,0]$} & {$[0,-1,0]$} & {$[0,1,0]$} & {$[0,0,0]$} & {$[0,0,0]$} & {$[0,0,0]$} & {$[0,0,0]$} \\
\hline & $\mathrm{Ag}_{3}(b)$ & {$[0,0,0]$} & {$[0,0,0]$} & {$[0,0,0]$} & {$[0,0,0]$} & {$[1,0,0]$} & {$[-1,0,0]$} & {$[-1,0,0]$} & {$[1,0,0]$} \\
\hline & $\operatorname{Ag}_{4}(a)$ & {$[0,0,0]$} & {$[0,0,0]$} & {$[0,0,0]$} & {$[0,0,0]$} & {$[1,0,0]$} & {$[1,0,0]$} & {$[1,0,0]$} & {$[1,0,0]$} \\
\hline & $\operatorname{Ag}_{4}(b)$ & {$[0,1,0]$} & {$[0,1,0]$} & {$[0,1,0]$} & {$[0,1,0]$} & {$[0,0,0]$} & {$[0,0,0]$} & {$[0,0,0]$} & {$[0,0,0]$} \\
\hline & $\operatorname{Ag}_{5}(a)$ & {$[0,0,1]$} & {$[0,0,-1]$} & {$[0,0,1]$} & {$[0,0,-1]$} & {$[0,0,0]$} & {$[0,0,0]$} & {$[0,0,0]$} & {$[0,0,0]$} \\
\hline & $\operatorname{Ag}_{5}(b)$ & {$[0,0,0]$} & {$[0,0,0]$} & {$[0,0,0]$} & {$[0,0,0]$} & {$[0,0,1]$} & {$[0,0,1]$} & {$[0,0,-1]$} & {$[0,0,-1]$} \\
\hline & $\operatorname{Ag}_{6}(a)$ & {$[0,0,0]$} & {$[0,0,0]$} & {$[0,0,0]$} & {$[0,0,0]$} & {$[0,0,1]$} & {$[0,0,-1]$} & {$[0,0,1]$} & {$[0,0,-1]$} \\
\hline & $\operatorname{Ag}_{6}(b)$ & {$[0,0,1]$} & {$[0,0,1]$} & {$[0,0,-1]$} & {$[0,0,-1]$} & {$[0,0,0]$} & {$[0,0,0]$} & {$[0,0,0]$} & {$[0,0,0]$} \\
\hline
\end{tabular}


TABLE VI. Crystal structure parameters of $\mathrm{Sm}_{2} \mathrm{Mn}\left(\mathrm{Mn}_{0.84} \mathrm{Sm}_{0.16}\right) \mathrm{Mn}_{3} \mathrm{TiO}_{12}\left(Z=2\right.$, space group $\left.P 4_{2} / n m c\right)$ refined at $80 \mathrm{~K}$. The lattice parameters were determined to be $a=7.4072(1) \AA$ and $c=7.9304(3) \AA$. Excellent reliability parameters of $R=2.72 \%, w R=2.90 \%$, and $R_{\mathrm{Bragg}}=4.06 \%$ were achieved in the refinement. Bond valence sums (BVSs) were calculated using the bond valence parameters $R_{0}\left(\mathrm{Sm}^{3+}\right)=$ 2.01(1), $R_{0}\left(\mathrm{Mn}^{3+}\right)=1.76(1), R_{0}\left(\mathrm{Mn}^{2+}\right)=1.79(1), R_{0}\left(\mathrm{Ti}^{4+}\right)=1.82(1)$, and $B=0.37$ [35]. N.B. the Wyckoff position of the Mn1 ions is listed for the split site with an occupation of 0.5 , and the high-symmetry position is given in square brackets with an occupation of 1 .

\begin{tabular}{lccccccc}
\hline \hline Ion & Site & Symmetry & $x$ & $y$ & $z$ & $U_{\text {iso }}\left(\AA^{2}\right)$ & BVS $(|e|)$ \\
\hline Sm1 & $4 d$ & $2 m m$. & 0.25 & 0.25 & $0.211(3)$ & $0.010(9)$ & $3.2(2)$ \\
Mn1 & $4 c[2 a]$ & $2 m m$. & 0.75 & 0.25 & $0.720(1)$ & $0.009(4)$ & $2.34(4)$ \\
Mn2 & $2 b$ & $\overline{4} m 2$ & 0.75 & 0.25 & 0.25 & $0.008(5)$ & $1.74(4)$ \\
Mn3/Ti1 & $8 e$ & $\overline{1}$ & 0 & 0 & 0 & $0.023(2)$ & $3.7(1) / 3.23(9)$ \\
O1 & $8 g$ &.$m$. & 0.25 & $0.0597(4)$ & $-0.0370(3)$ & $0.028(2)$ & $0.028(2)$ \\
O2 & $8 g$ &.$m$. & 0.25 & $0.5356(4)$ & $0.5770(4)$ & $0.029(2)$ \\
O3 & $8 f$ &. .2 & $0.4355(2)$ & $-0.4355(2)$ & 0.25 & \\
\hline \hline
\end{tabular}

TABLE VII. Crystal structure parameters of $\mathrm{Sm}_{2} \mathrm{Mn}\left(\mathrm{Mn}_{0.93} \mathrm{Sm}_{0.07}\right) \mathrm{Mn}_{2} \mathrm{Ti}_{2} \mathrm{O}_{12}\left(Z=2\right.$, space group $\left.P 4_{2} / n m c\right)$ refined at $90 \mathrm{~K}$. The lattice parameters were determined to be $a=7.5506(1) \AA$ and $c=7.7273(3) \AA$. Excellent reliability parameters of $R=2.71 \%, w R=2.87 \%$, and $R_{\text {Bragg }}=5.05 \%$ were achieved in the refinement. Bond valence sums were calculated using the bond valence parameters $R_{0}\left(\operatorname{Sm}{ }^{3+}\right)=2.01(1)$, $R_{0}\left(\mathrm{Mn}^{2+}\right)=1.79(1), R_{0}\left(\mathrm{Mn}^{3+}\right)=1.76(1), R_{0}\left(\mathrm{Ti}^{4+}\right)=1.82(1)$, and $B=0.37$ [35]. N.B. the Wyckoff position of the Mn1 ions is listed for the split site with an occupation of 0.5 , and the high-symmetry position is given in square brackets with an occupation of 1 .

\begin{tabular}{lccccccc}
\hline \hline Ion & Site & Symmetry & $x$ & $y$ & $z$ & $U_{\text {iso }}\left(\AA^{2}\right)$ & BVS $(|e|)$ \\
\hline Sm1 & $4 d$ & $2 m m$. & 0.25 & 0.25 & $0.209(3)$ & $0.022(4)$ & $3.2(2)$ \\
Mn1 & $4 c[2 a]$ & $2 m m$. & 0.75 & 0.25 & $0.789(1)$ & $0.022(4)$ & $1.78(5)$ \\
Mn2 & $2 b$ & $\overline{4} m 2$ & 0.75 & 0.25 & 0.25 & $0.022(4)$ & $1.71(4)$ \\
Mn3/Ti1 & $8 e$ & $\overline{1}$ & 0 & 0 & 0 & $0.014(2)$ & $3.7(1) / 3.22(9)$ \\
O1 & $8 g$ &.$m$. & 0.25 & $0.0611(4)$ & $-0.0343(3)$ & $0.019(2)$ & $0.022(2)$ \\
O2 & $8 g$ &.$m$. & 0.25 & $0.5406(4)$ & $0.5705(4)$ & $0.023(2)$ \\
O3 & $8 f$ &. .2 & $0.4436(2)$ & $-0.4436(2)$ & 0.25 & \\
\hline \hline
\end{tabular}

TABLE VIII. Crystal structure parameters of $\mathrm{Sm}_{2} \mathrm{MnMnMnTi}_{3} \mathrm{O}_{12}\left(Z=2\right.$, space group $\left.P 4_{2} / n m c\right)$ refined at $80 \mathrm{~K}$. The lattice parameters were determined to be $a=7.6290(1) \AA$ and $c=7.6932(3) \AA$. Excellent reliability parameters of $R=2.03 \%, w R=2.20 \%$, and $R_{\text {Bragg }}=5.69 \%$ were achieved in the refinement. Bond valence sums were calculated using the bond valence parameters $R_{0}\left(\operatorname{Sm}^{3+}\right)=2.01(1)$, $R_{0}\left(\mathrm{Mn}^{2+}\right)=1.79(1), R_{0}\left(\mathrm{Mn}^{3+}\right)=1.76(1), R_{0}\left(\mathrm{Ti}^{4+}\right)=1.82(1)$, and $B=0.37$ [35]. N.B. the Wyckoff position of the Mn1 ions is listed for the split site with an occupation of 0.5 , and the high-symmetry position is given in brackets with an occupation of 1 .

\begin{tabular}{|c|c|c|c|c|c|c|c|}
\hline Ion & Site & Symmetry & $x$ & $y$ & $z$ & $U_{\text {iso }}\left(\AA^{2}\right)$ & $\operatorname{BVS}(|e|)$ \\
\hline Sm1 & $4 d$ & $2 m m$. & 0.25 & 0.25 & $0.214(3)$ & $0.023(3)$ & $3.0(2)$ \\
\hline Mn1 & $4 c[2 a]$ & $2 \mathrm{~mm}$. & 0.75 & 0.25 & $0.797(1)$ & $0.023(3)$ & $1.58(2)$ \\
\hline Mn3/Ti1 & $8 e$ & $\overline{1}$ & 0 & 0 & 0 & $0.022(2)$ & $3.6(1) / 3.36(9)$ \\
\hline $\mathrm{O} 1$ & $8 g$ &.$m$ & 0.25 & $0.0641(4)$ & $-0.0339(4)$ & $0.027(2)$ & \\
\hline $\mathrm{O} 2$ & $8 g$ & $m$ & 0.25 & $0.5441(4)$ & $0.5719(3)$ & $0.024(2)$ & \\
\hline
\end{tabular}

[1] S. Jin, T. H. Tiefel, M. McCormack, R. Fastnacht, R. Ramesh, and L. Chen, Science 264, 413 (1994).

[2] E. O. Wollan and W. C. Koehler, Phys. Rev. 100, 545 (1955).
[3] J.-S. Zhou and J. B. Goodenough, Phys. Rev. Lett. 96, 247202 (2006).

[4] P. G. Radaelli, D. E. Cox, M. Marezio, and S.-W. Cheong, Phys. Rev. B 55, 3015 (1997). 
[5] J. B. Goodenough, Phys. Rev. 100, 564 (1955).

[6] A. Muñoz, M. T. Casáis, J. A. Alonso, M. J. Martínez-Lope, J. L. Martínez, and M. T. Fernández-Díaz, Inorg. Chem. 40, 1020 (2001).

[7] A. M. Glazer, Acta Crystallogr., Sect. B: Struct. Crystallogr. Cryst. Chem. 28, 3384 (1972).

[8] A. A. Belik, Y. S. Glazkova, Y. Katsuya, M. Tanaka, A. V. Sobolev, and I. A. Presniakov, J. Phys. Chem. C 120, 8278 (2016).

[9] L. Zhang, N. Terada, R. D. Johnson, D. D. Khalyavin, P. Manuel, Y. Katsuya, M. Tanaka, Y. Matsushita, K. Yamaura, and A. A. Belik, Inorg. Chem. 57, 5987 (2018).

[10] R. D. Johnson, D. D. Khalyavin, P. Manuel, L. Zhang, K. Yamaura, and A. A. Belik, Phys. Rev. B 98, 104423 (2018).

[11] R. D. Johnson, D. D. Khalyavin, P. Manuel, Y. Katsuya, M. Tanaka, Y. Matsushita, L. Zhang, K. Yamaura, and A. A. Belik, Phys. Rev. B 99, 024107 (2019).

[12] R. D. Johnson, D. D. Khalyavin, P. Manuel, A. Bombardi, C. Martin, L. C. Chapon, and P. G. Radaelli, Phys. Rev. B 93, 180403(R) (2016).

[13] R. D. Johnson, D. D. Khalyavin, P. Manuel, P. G. Radaelli, I. S. Glazkova, N. Terada, and A. A. Belik, Phys. Rev. B 96, 054448 (2017)

[14] R. D. Johnson, F. Mezzadri, P. Manuel, D. D. Khalyavin, E. Gilioli, and P. G. Radaelli, Phys. Rev. Lett. 120, 257202 (2018)

[15] L. Zhang, Y. Matsushita, K. Yamaura, and A. A. Belik, Inorg. Chem. 56, 5210 (2017).

[16] R. Liu, R. Scatena, D. D. Khalyavin, R. D. Johnson, Y. Inaguma, M. Tanaka, Y. Matsushita, K. Yamaura, and A. A. Belik, Inorg. Chem. 59, 9065 (2020).

[17] A. M. Vibhakar, D. D. Khalyavin, P. Manuel, L. Zhang, K. Yamaura, P. G. Radaelli, A. A. Belik, and R. D. Johnson, Phys. Rev. B 99, 104424 (2019).
[18] A. M. Vibhakar, D. D. Khalyavin, P. Manuel, J. Liu, A. A. Belik, and R. D. Johnson, Phys. Rev. Lett. 124, 127201 (2020).

[19] A. A. Belik, L. Zhang, R. Liu, D. D. Khalyavin, Y. Katsuya, M. Tanaka, and K. Yamaura, Inorg. Chem. 58, 3492 (2019).

[20] R. J. Elliott and B. Heap, Proc. R. Soc. London, Ser. A 265, 264 (1962).

[21] L. C. Chapon, P. Manuel, P. G. Radaelli, C. Benson, L. Perrott, S. Ansell, N. J. Rhodes, D. Raspino, D. Duxbury, E. Spill, and J. Norris, Neutron News 22, 22 (2011).

[22] B. J. Campbell, H. T. Stokes, D. E. Tanner, and D. M. Hatch, J. Appl. Crystallogr. 39, 607 (2006).

[23] J. Rodríguez-Carvajal, Phys. B: Condens. Matter 192, 55 (1993).

[24] M.-H. Whangbo, E. E. Gordon, H. Xiang, H.-J. Koo, and C. Lee, Acc. Chem. Res. 48, 3080 (2015).

[25] R. L. White, J. Appl. Phys. (Melville, NY) 40, 1061 (1969).

[26] M. Hutchings, Solid State Phys. 16, 227 (1964).

[27] D. Stauffer and A. Aharony, Introduction to Percolation Theory, 2nd ed. (Taylor \& Francis, London, 1985).

[28] D. P. Landau, Phys. Rev. B 22, 2450 (1980).

[29] A. Zvyagin and A. Anders, J. Exp. Theor. Phys. 40, 154 (1975).

[30] S.-W. Cheong, A. S. Cooper, L. W. Rupp, B. Batlogg, J. D. Thompson, and Z. Fisk, Phys. Rev. B 44, 9739 (1991).

[31] J. F. Niven, M. B. Johnson, A. Bourque, P. J. Murray, D. D. James, H. A. Dabkowska, B. D. Gaulin, and M. A. White, Proc. R. Soc. A 470, 20140387 (2014).

[32] J. H. Van Vleck, Rev. Mod. Phys. 17, 27 (1945).

[33] J. Sakurai, W. J. L. Buyers, R. A. Cowley, and G. Dolling, Phys. Rev. 167, 510 (1968).

[34] M. Janoschek, B. Roessli, L. Keller, S. N. Gvasaliya, K. Conder, and E. Pomjakushina, J. Phys.: Condens. Matter 17, L425 (2005).

[35] N. E. Brese and M. O'Keeffe, Acta Crystallogr., Sect. B: Struct. Sci. 47, 192 (1991). 